\title{
Zinc oxide nanorod array as an inhibitory biointerface
}

\author{
Yongchen Wang, Department of Biomedical Engineering, The Ohio State University, Columbus 43210, USA \\ Jordan D. Prox, Biomedical Sciences Graduate Program, The Ohio State University, Columbus 43210, USA \\ Bingxi Yan, and Yu Wu, Department of Electrical and Computer Engineering, The Ohio State University, Columbus 43210, USA \\ Aaron D. Argall, Biomedical Sciences Graduate Program, The Ohio State University, Columbus 43210, USA \\ Liang Guo, Department of Electrical and Computer Engineering, The Ohio State University, Columbus 43210, USA; Department of Neuroscience, The Ohio \\ State University, Columbus 43210, USA \\ Address all correspondence to Liang Guo at guo.725@osu.edu
}

(Received 30 May 2018; accepted 28 August 2018)

\section{Abstract}

One-dimensional zinc oxide ( $\mathrm{ZnO}$ ) nanostructure arrays show unique semiconducting, piezoelectric, and wetting properties, and how they interact with cells is critical for their biomedical applications. In this work, we prepare ZnO nanorod arrays (ZnO NRAs) and study their interactions with neonatal rat cardiomyocytes either as a substrate or patch. We find that ZnO NRAs can (1) inhibit cell adhesion and spreading as a substrate and (2) selectively kill underneath cells as a patch. We further identify surface nanomorphology as the dominant factor responsible for the inhibitory effect. These discoveries suggest potential application of ZnO NRAs as a cell inhibitory biointerface.

\section{Introduction}

Zinc oxide $(\mathrm{ZnO})$ nanomaterials exhibit unique semiconducting, piezoelectric, and surface wetting properties, and they have been widely studied for sensors, transistors, optoelectronics, generators, consumer goods, etc. ${ }^{[1-3]}$ They have also been investigated for biomedical applications, such as selective cancer cell destruction, drug delivery, and antibiotics. ${ }^{[4,5]}$ Compared to dispersed nanomaterials, arrays of onedimensional (1D) $\mathrm{ZnO}$ nanostructures, such as nanowires, nanorods, and nanobelts, present unique surface topography and large surface area, enable high outputs, and are retrievable, ${ }^{[6]}$ so they are of particular interest for biomedical applications.

For their biomedical applications, it is crucial to scrutinize how they interact with cells. 1D $\mathrm{ZnO}$ nanostructure arrays showed good biocompatibility. Cells showed good adhesion, proliferation, differentiation, and viability when interacting with 1D $\mathrm{ZnO}$ nanostructure arrays. ${ }^{[7-12]}$ However, 1D $\mathrm{ZnO}$ nanostructure arrays also induced inhibitory effects on cells, decreasing cell adhesion and viability and inhibiting differentiation. ${ }^{[8,9,12-15]}$ Thus, it is critical to fully understand the mechanism so that we can modulate cellular responses in a controlled manner.

The biological effects of $1 \mathrm{D} \mathrm{ZnO}$ nanostructure arrays can depend on many factors. First, the nanomorphology matters. $\mathrm{ZnO}$ nanorod arrays ( $\mathrm{ZnO}$ NRAs) can have different diameters, spacings, lengths, densities, shapes, and alignments, and all can impact cellular responses. Second, it depends on cell type. For example, cell lines respond differently than primary cells, ${ }^{[4,12,16]}$ and different cell lines respond differently as well. ${ }^{[8,15]}$ Additionally, if ZnO's surface chemistry leads to production of reactive oxygen species ${ }^{[17]}$ and/or $\mathrm{Zn}^{2+}$ dissolution, ${ }^{[14]}$ if cells sense piezoelectricity, ${ }^{[10]}$ and if $1 \mathrm{D} \mathrm{ZnO}$ nanostructure arrays interact with cells as a substrate or patch, could all affect the biological effects. In this work, we studied the biological effects of vertically aligned $\mathrm{ZnO}$ NRAs on primary cardiomyocytes of high metabolic demands by applying them as both a substrate and patch. The substrate method is to study the cell adhesion and spreading on $\mathrm{ZnO}$ NRAs, and the patch method is to mimic the intimate interfacing within a constrained environment when applied in tissues.

\section{Materials and methods Preparation of ZnO NRAs and gold (Au)-coated ZnO NRAs}

All chemicals were purchased from Sigma-Aldrich (Saint Louis, MO, USA) and used without purification. ZnO NRAs were synthesized via a simple two-step seedless hydrothermal method. ${ }^{[18,19]}$ Briefly, in the first step, $10 \mathrm{~nm}$ thick titanium (Ti) and $50 \mathrm{~nm}$ thick Au were sequentially deposited onto $18 \mathrm{~mm}$-diameter circular cover glasses at a deposition rate of $1 \AA /$ s using an electron-beam evaporator (DV-502A, Denton Vacuum). Au-coated cover glasses were annealed at $300{ }^{\circ} \mathrm{C}$ for $1 \mathrm{~h}$ in a furnace (62700, Barnstead Thermolyne). In the second step, Au-coated cover glasses were gently placed to float on an aqueous reaction solution $(12.5 \mathrm{mM}$ hexamethylenetetramine, $25 \mathrm{mM}$ zinc nitrate hexahydrate, and $0.70 \mathrm{M}$ 
ammonium hydroxide) with the Au-coated side facing the solution. The reaction proceeded at $80{ }^{\circ} \mathrm{C}$ for $24 \mathrm{~h}$ in an oven (Gravity Ovens, Fisher Scientific). ZnO NRAs grown on Au-coated cover glasses were thoroughly washed with distilled water and air dried. Au-coated $\mathrm{ZnO}$ NRAs were acquired by depositing $10 \mathrm{~nm}$ thick $\mathrm{Ti}$ and $50 \mathrm{~nm}$ thick $\mathrm{Au}$ onto the $\mathrm{ZnO}$ NRAs using the deposition method above.

\section{Surface characterization using a scanning electron microscope (SEM), an atomic force microscope (AFM), a contact angle goniometer, and X-ray photoelectron spectroscopy (XPS)}

The surface morphologies of $\mathrm{ZnO}$ NRAs and Au-coated $\mathrm{ZnO}$ NRAs were imaged using an SEM (Nova NanoSEM 400, FEI). The SEM images were processed and analyzed using Image J (National Institutes of Health). The surface morphology of $\mathrm{ZnO}$ NRAs was further studied using an AFM (Dimension Icon, Bruker) in a soft tapping mode with a silicon probe (RTESPA-150, Bruker) having a resonant frequency of $150 \mathrm{kHz}$ and a spring constant of $6 \mathrm{~N} / \mathrm{m}$. The AFM images were processed and analyzed using NanoScope Analysis (Bruker). The surface wettability of $\mathrm{ZnO}$ NRAs, Au-coated $\mathrm{ZnO}$ NRAs, and Au-coated cover glasses was studied with a standard contact angle goniometer (200-F1, ramé-hart), and the contact angle images and data were acquired with Dropimage Advanced software (ramé-hart). The surface chemistry of $\mathrm{ZnO}$ NRAs, Au-coated ZnO NRAs, and Au-coated cover glasses were studied using XPS (Axis Ultra, Kratos Analytical), and the XPS spectra were analyzed using CasaXPS (Casa Software Ltd.). All peak positions were calibrated by keeping the binding energy of $\mathrm{C} 1 \mathrm{~s}$ at 285 eV. ${ }^{[20,21]}$

\section{Cardiomyocyte harvesting and culture}

The protocol for harvesting ventricular cardiac muscle cells from the hearts of post-natal day 2 Sprague-Dawley rat pups was adopted from Richard Pattern at Tufts Medical Center, Boston, MA, USA ${ }^{[22]}$ and approved by the Institutional Animal Care and Use Committee at The Ohio State University, Columbus, OH, USA. Briefly, great vessels and atria were trimmed from the ventricles of each heart, and then ventricles were placed in a dissociation buffer $(116 \mathrm{mM}$ $\mathrm{NaCl}, 5.4 \mathrm{mM} \mathrm{KCl}, 0.8 \mathrm{mM} \mathrm{MgSO}$, $0.8 \mathrm{mM} \mathrm{Na} \mathrm{HPO}_{4}, 20$ $\mathrm{mM}$ HEPES, and 5.6 mM glucose, $\mathrm{pH} 7.35$ ). Ventricles were minced with forceps and micro-scissors and then dissociated through a serial enzymatic digestion (the previous dissociation buffer supplemented with $0.06 \%$ pancreatin and $0.04 \%$ collagenase type II) at $37^{\circ} \mathrm{C}$. Cell suspensions were collected and placed in neonatal calf serum after each digestion. Cell suspensions were then pooled and re-suspended in a culture medium (Dulbecco's modified Eagle's medium supplemented with $10 \%$ horse serum, $7 \%$ charcoal-stripped fetal bovine serum, $100 \mathrm{U} / \mathrm{L}$ penicillin, and $100 \mu \mathrm{g} / \mathrm{mL}$ streptomycin). The suspension was passed through a $70 \mu \mathrm{m}$ pore-sized nylon filter and pre-plated for $75 \mathrm{~min}$. The cardiomyocyte-enriched fraction was then removed and plated as needed. Cells were cultured in the culture medium in an incubator under $95 \%$ air and $5 \%$ $\mathrm{CO}_{2}$ at $37^{\circ} \mathrm{C}$.

\section{Substrate method studied by F-actin and 4',6-diamidino-2-phenylindole (DAPI) staining}

The substrates studied included $\mathrm{ZnO}$ NRAs, Au-coated $\mathrm{ZnO}$ NRAs, Au-coated cover glasses, and polystyrene (bottom surface material of culture plate wells). On Day 0, substrates were immersed in 70\% (v/v) ethanol for $15 \mathrm{~min}$ for decontamination, thoroughly washed with phosphate-buffered saline (PBS), and coated with $25 \mu \mathrm{g} / \mathrm{mL}$ fibronectin overnight in the incubator. On Day 1, the fibronectin-coated substrates were washed with distilled water and air dried. Each substrate was placed in a well of a 12-well culture plate. Cardiomyocytes were harvested, and $10^{5}$ cells in $1 \mathrm{~mL}$ of culture medium was added per well. On Day 2, after the cells were cultured for 1 day, cardiomyocytes were stained with a fluorescent F-actin probe (ActinRed ${ }^{\mathrm{TM}} 555$ ReadyProbes $^{\mathrm{TM}}$ Reagent, ThermoFisher Scientific) and DAPI. Briefly, cells were washed with Dulbecco's PBS (DPBS) and fixed with 4\% paraformaldehyde for $30 \mathrm{~min}$ at room temperature. Cells were again washed with DPBS and stained with the F-actin probe solution in DPBS $(9 \%(\mathrm{v} / \mathrm{v})$ F-actin probe reagent and $0.5 \%(\mathrm{v} / \mathrm{v})$ Triton $\mathrm{X}-100$ ) for $30 \mathrm{~min}$ at room temperature in the dark. Cells were finally washed with DPBS and then stained with a mounting medium containing DAPI (Fluoromount-G ${ }^{\mathrm{TM}}$, ThermoFisher Scientific). The stained cells were imaged with an inverted fluorescence microscope (EVOS, ThermoFisher Scientific). Images were processed using ImageJ.

\section{Patch method studied by live/dead staining with fluorescein diacetate (FDA) and propidium iodide (PI)}

The patches studied included $\mathrm{ZnO}$ NRAs, Au-coated $\mathrm{ZnO}$ NRAs, and Au-coated cover glasses, and polystyrene served as an unpatched control. On Day 0, a 12-well culture plate was coated with $25 \mu \mathrm{g} / \mathrm{mL}$ fibronectin overnight in the incubator. On Day 1, the culture plate was washed with distilled water and air dried. Cardiomyocytes were harvested, and $2 \times 10^{5}$ cells in $1 \mathrm{~mL}$ of culture medium was added per well. On Day 2, after cells were cultured for 1 day, materials were patched. Briefly, patches were immersed in $70 \%(\mathrm{v} / \mathrm{v})$ ethanol for $15 \mathrm{~min}$ for decontamination, thoroughly washed with PBS, and air dried. Then, the materials were gently placed as patches to loosely contact the cells on the well bottom with the materials facing the cells. On Day 3, patches were retrieved, and live/dead staining was conducted, ${ }^{[23]}$ in which live cells were stained to green with FDA, and dead cells were stained to red with PI. $2 \times 10^{5}$ cells were stained with $333.3 \mathrm{ng}$ FDA and $100 \mathrm{ng}$ PI for $5 \mathrm{~min}$ at room temperature in the dark. The stained cells were washed with DPBS and imaged with a fluorescence microscope. Images were processed using ImageJ. 


\section{Results}

Synthesis and surface characterization of $\mathrm{ZnO}$ NRAs, Au-coated ZnO NRAs, and Au-coated cover glasses

As shown in the SEM image in Fig. 1(a), a vertically aligned and densely packed $\mathrm{ZnO}$ NRA was grown on an Au-coated cover glass. In the SEM image of an Au-coated ZnO NRA in Fig. 1(b), the $10 \mathrm{~nm}$ thick $\mathrm{Ti}$ and $50 \mathrm{~nm}$ thick Au coatings did not alter the array morphology. Measured from these SEM images, the $\mathrm{ZnO}$ nanorods had a diameter of approximately $250 \mathrm{~nm}$, and the Au-coated $\mathrm{ZnO}$ nanorods had a diameter of approximately $290 \mathrm{~nm}$. The array morphology of $\mathrm{ZnO}$ NRAs was further confirmed and studied by an AFM. From the two-dimensional (2D) [Fig. 1(c)] and three-dimensional (3D) [Fig. 1(d)] height maps, a vertically aligned and densely packed array could be observed. The wettability of the materials was studied with a contact angle goniometer. The water contact angle (WCA) on an Au-coated cover glass was $122.13^{\circ} \pm$ $5.47^{\circ}$, and the WCA on an Au-coated ZnO NRA was $124.48^{\circ} \pm$ $4.51^{\circ}$. Water droplets spread quickly on ZnO NRAs.

The surface chemistry of the materials was studied with XPS. As seen in the survey spectra, the surface chemistry of a ZnO NRA [Fig. 1(e)] contains zinc ( $\mathrm{Zn})$, oxygen $(\mathrm{O})$, and adventitious carbon (C); the surface chemistry of an Au-coated $\mathrm{ZnO}$ NRA [Fig. 1(f)] contains gold, oxygen, adventitious carbon, and trace of zinc; the surface of an Au-coated cover glass [Fig. 1(g)] contains gold, oxygen, and adventitious carbon. The specific percentage atomic concentrations are listed in Table I. The high-resolution spectrum of $\mathrm{Zn}$ $2 \mathrm{p}$ [Fig. S1(a)] in $\mathrm{ZnO}$ shows its characteristic peaks of $\mathrm{Zn} 2 \mathrm{p}_{1 / 2}$ at $1044.9 \mathrm{eV}$ and $\mathrm{Zn} 2 \mathrm{p}_{3 / 2}$ at $1021.8 \mathrm{eV} .^{[24,25]}$ The highresolution spectrum of $\mathrm{O} 1 \mathrm{~s}$ [Fig. $\mathrm{S} 1(\mathrm{~b})]$ in $\mathrm{ZnO}$ was deconvoluted to the oxygen from $\mathrm{ZnO}$ at $530.4 \mathrm{eV}$ and chemisorbed oxygen at $531.8 \mathrm{eV} \cdot{ }^{[26,27]}$ The high-resolution spectra of $\mathrm{Au} 4 \mathrm{f}$ in an Au-coated ZnO NRA [Fig. S1(c)] and an Au-coated cover glass [Fig. S1(d)] show similar peaks. These results indicate that $\mathrm{ZnO}$ NRAs are prepared and that an Au-coated ZnO NRA and an Au-coated cover glass have similar surface chemistry.

\section{Substrate method}

As shown in Fig. 2(a), the material (diameter: $18 \mathrm{~mm}$ ) was placed facing upward as a substrate in a single well of a 12-well plate (well diameter: $22.6 \mathrm{~mm}$ ), leaving a surrounding ring that was not covered by the substrate. Both Areas 1 and 2 were imaged, and their fluorescent images are shown in Fig. 3 and Fig. S2, respectively. In these images, the actin filaments in the cytoskeleton were stained to red with a fluorescent F-actin probe, and the nuclei were stained to blue with DAPI.

As seen in Fig. 3, at the central area of the substrate (Area 1), cells well attached, spread, and became almost fully confluent (a)
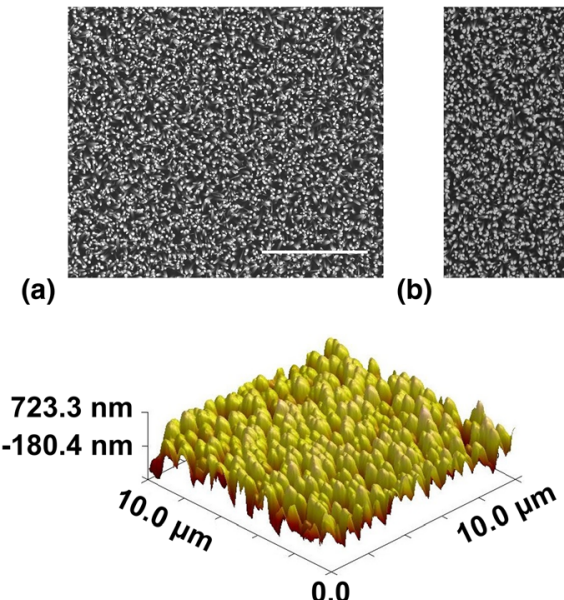

(d)

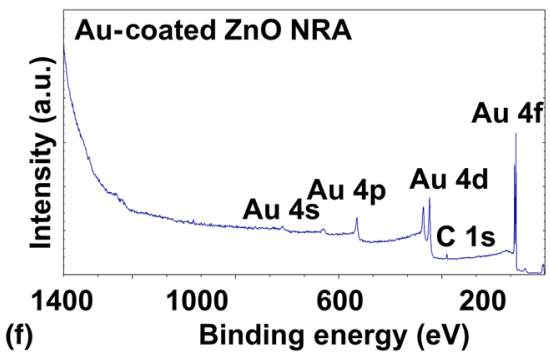

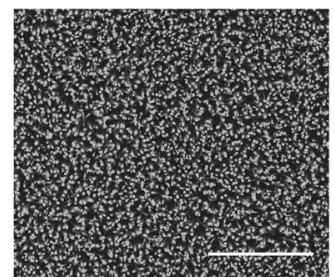

(c) 0.0

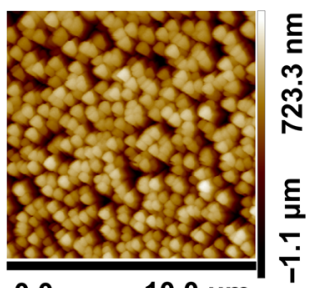

$10.0 \mu \mathrm{m}$
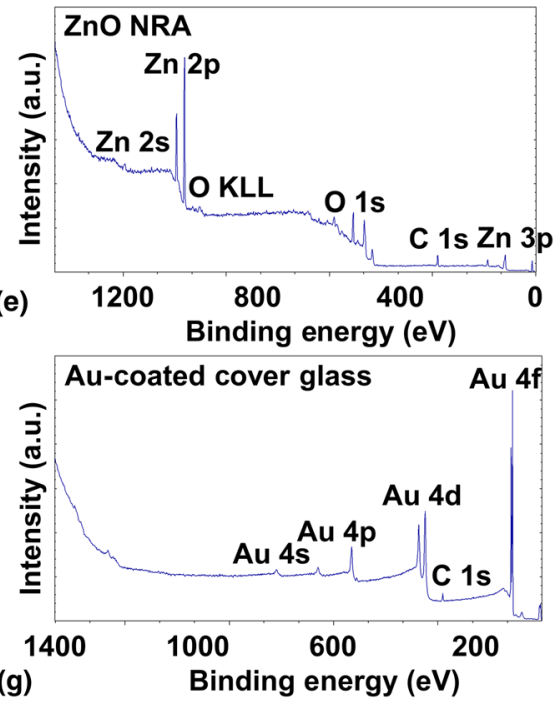

Figure 1. Material characterization. (a) An SEM image of a ZnO NRA. Scale bar: $10 \mu \mathrm{m}$. (b) An SEM image of an Au-coated ZnO NRA. Scale bar: $10 \mu \mathrm{m}$ (c) A 2D AFM height map of a ZnO NRA. Scanning size: $10 \mu \mathrm{m} \times 10 \mu \mathrm{m}$. (d) A 3D AFM height map of a ZnO NRA. Scanning size: $10 \mu \mathrm{m} \times 10 \mu \mathrm{m}$. (e) The XPS survey spectrum of a ZnO NRA. (f) The XPS survey spectrum of an Au-coated ZnO NRA. (g) The XPS survey spectrum of an Au-coated cover glass. 
Table I. Percentage atomic concentrations of a ZnO NRA, an Au-coated ZnO NRA, and an Au-coated cover glass.

\begin{tabular}{llll}
\hline & \multicolumn{3}{c}{ Percentage atomic concentration (\%) } \\
\cline { 2 - 4 } Regions & ZnO NRA & $\begin{array}{c}\text { Au-coated } \\
\text { ZnO NRA }\end{array}$ & $\begin{array}{c}\text { Au-coated } \\
\text { cover glass }\end{array}$ \\
\hline Zn 2p & 21.42 & 0.25 & 0 \\
\hline \multirow{2}{*}{ 0 1s } & $17.27(530.4 \mathrm{eV})$ & \multirow{2}{*}{3.09} & 2.72 \\
\cline { 2 - 3 } & $20.98(531.8 \mathrm{eV})$ & & 24.62 \\
\hline C 1s & 40.33 & 29.14 & 72.66 \\
\hline Au 4f & 0 & 67.52 & \\
\hline
\end{tabular}

on polystyrene and an Au-coated cover glass. However, on the $\mathrm{ZnO}$ and $\mathrm{Au}$-coated $\mathrm{ZnO}$ NRAs, majority of the cells showed limited adhesion and exhibited a spherical shape, with only a small percentage attaching to the substrates and displaying an elongated morphology. As seen in Fig. S2, at the edge area of the well not covered by the substrate (Area 2), cells well attached and spread in all experimental groups.

\section{Patch method}

After being cultured for 1 day, the cells attached and spread well (Fig. S3). As shown in Fig. 2(b), the material (diameter: $18 \mathrm{~mm}$ ) was placed facing downward as a patch on the attached cells in a single well of a 12-well plate (well diameter: 22.6 $\mathrm{mm}$ ), leaving a surrounding unpatched ring. Both Areas 3 and 4 were imaged, and their fluorescent images are shown in Fig. 4 and Fig. S4, respectively. In these images, live cells were stained to green with FDA, and dead cells were stained to red with PI.

As seen in Fig. 4 and Fig. S5(a), at the central area (Area 3), the cells cultured on polystyrene and patched by an Au-coated cover glass showed a high viability. In contrast, almost all cells patched by the $\mathrm{ZnO}$ and $\mathrm{Au}$-coated $\mathrm{ZnO}$ NRAs died. As seen in Fig. S4 and Fig. S5(b), at the boundary region (Area 4), there was no obvious viability difference along the boundary for polystyrene and an Au-coated cover glass. However, for the $\mathrm{ZnO}$ and $\mathrm{Au}$-coated $\mathrm{ZnO}$ NRAs, there was a clearly defined boundary, along which the cells closer to the center were all dead, but the cells further away from the center showed a high viability.

\section{Discussion and outlook}

In this work, we studied vertically aligned and densely packed $\mathrm{ZnO}$ NRAs as either a substrate or patch to interface with primary neonatal rat cardiomyocytes. We found that, when used as a substrate, $\mathrm{ZnO}$ NRAs inhibited cell adhesion, causing majority of the cells to assume a spherical shape; and, when used as a patch, $\mathrm{ZnO}$ NRAs killed majority of the patched cells underneath without affecting the surrounding cells. Therefore, our results indicate that ZnO NRAs have an inhibitory effect on the attachment, spreading, and growth/survival of neonatal rat cardiomyocytes. Furthermore, a ZnO NRA and an Au-coated $\mathrm{ZnO}$ NRA have similar surface morphology but different surface chemistry, while an Au-coated ZnO NRA and an Au-coated cover glass have similar surface chemistry but different surface morphology. Based on our experimental evidence that the chemically inert control of an Au-coated $\mathrm{ZnO}$ NRA had the same inhibitory effects as a ZnO NRA and that an Au-coated cover glass showed the same biological effects as polystyrene, we can rule out the possibility of a chemical cause through the production of reactive oxygen species and dissolution of $\mathrm{Zn}^{2+}$. By comparing the surface wettability and biological effects, we can also rule out the wettability as a major factor. Thus, we conclude that the inhibitory effects are due to the nanomorphology.

When interacting with cells, 1D $\mathrm{ZnO}$ nanostructure arrays showed either biocompatibility ${ }^{[7-12]}$ or induced inhibitory effects. $^{[8,9,12-15]}$ The different biological effects can be due to the different surface morphologies (diameters, spacings, lengths, densities, shapes, and alignments) and cell types in different studies. Thus, the biological effects of $1 \mathrm{D} \mathrm{ZnO}$ nanostructure arrays need to be studied case by case. In this study, ZnO NRAs (with or without Au-coating) as substrates inhibit cell adhesion and spreading through its rough and discontinuous surface nanomorphology. The large spacing of

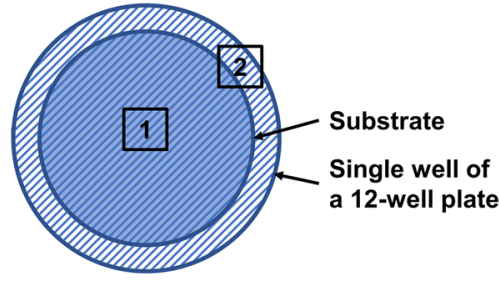

(a)

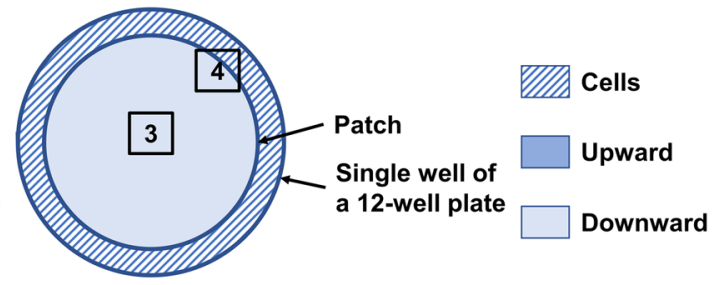

(b)

Figure 2. Illustration of the substrate and patch methods. The material (diameter: $18 \mathrm{~mm}$ ) was applied as either a substrate or patch in a single well of a 12-well culture plate (well diameter: $22.6 \mathrm{~mm}$ ). (a) In the substrate method, the material was first placed facing upward as a substrate, and cells were then plated. Areas 1 and 2 were imaged and shown in Fig. 3 and Fig. S2, respectively. (b) In the patch method, cells were first plated and cultured for 1 day, and the material was then placed facing downward as a patch. Areas 3 and 4 were imaged and shown in Fig. 4 and Fig. S4, respectively. 

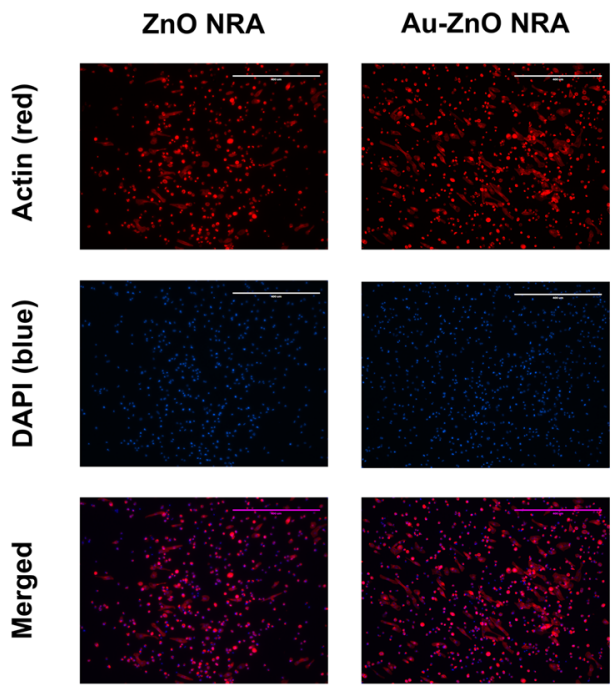
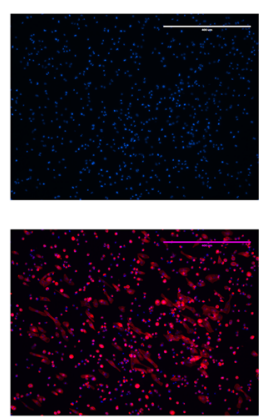

Au-GL
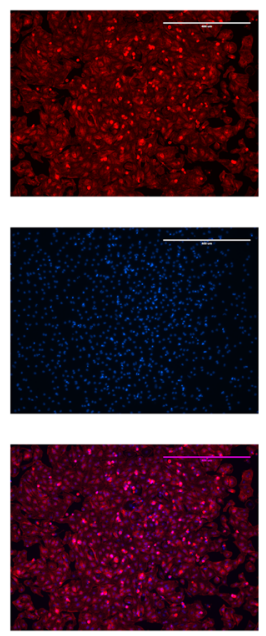

PS
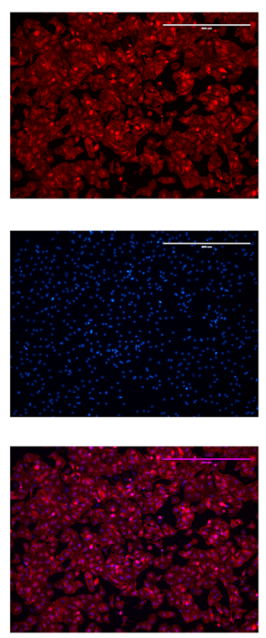

Figure 3. Fluorescent images of cardiomyocytes cultured on different substrates at the central area [Area 1 indicated in Fig. 2(a)]. The actin filaments in the cytoskeleton were stained to red with an actin probe (first row), and the nuclei were stained to blue with DAPI (second row). Third row (merged images of first and second rows) shows that cells well attached and spread on polystyrene and an Au-coated cover glass, while cells showed limited adhesion on the ZnO and Au-coated ZnO NRAs. Magnification: 10x. Scale bar: $400 \mu \mathrm{m}$. Abbreviations: Au-coated ZnO NRA (Au-ZnO NRA), Au-coated cover glass (Au-GL), and polystyrene (PS).

several hundred nanometers between nanorods could inhibit the formation of lamellipodia and focal adhesion. ${ }^{[9,13]}$ Interestingly, when interacting with $1 \mathrm{D} \mathrm{ZnO}$ nanostructure arrays, fast-dividing cell lines with inhibited adhesion eventually died, ${ }^{[12,13,15]}$ whereas primary cells with inhibited adhesion remained viable. ${ }^{[12,16]}$

The cause to cell death underneath ZnO NRAs (with or without Au-coating) should also be physical. Au-coated cover glasses were probably spaced by a thin solution layer from the cells, which explains the survival of the underneath cells. In contrast, $\mathrm{ZnO}$ NRAs (with or without Au-coating) could exclude such a thin solution layer from underneath by allowing the solution to fill the spaces between the nanorods as a result of the capillary effect ${ }^{[28,29]}$ and lead to a more intimate contact to the cell monolayer. In such a case, the diffusions of nutrients and oxygen should be very limited, and the cells underneath would die.

While further long-term study is needed, the discoveries here suggest potential application of this type of nanomaterial as a cell adhesion resistant coating for medical device. Given
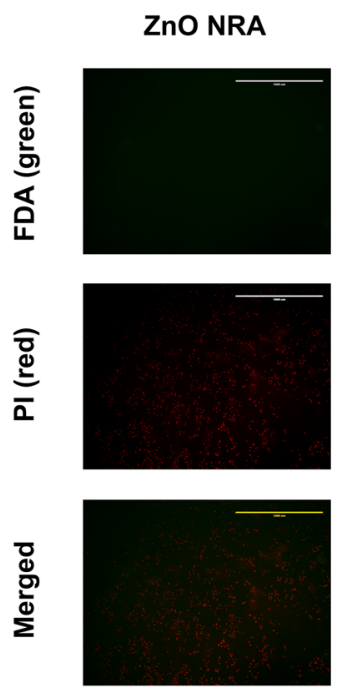

Au-ZnO NRA
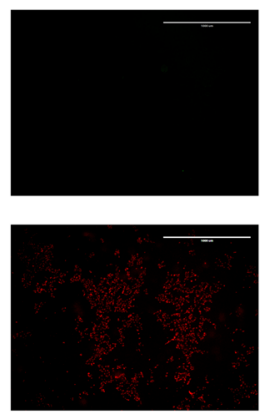

Au-GL
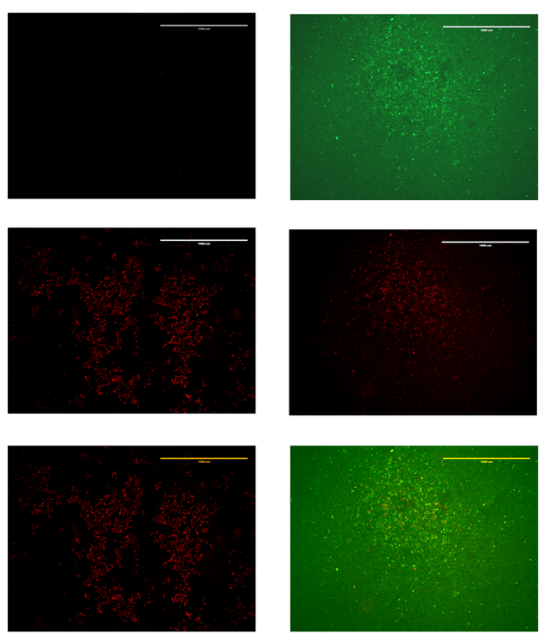

PS
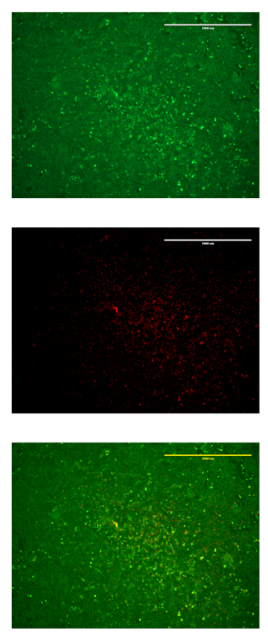

Figure 4. Fluorescent images of cardiomyocytes patched by different materials at the central area [Area 3 indicated in Fig. 2(b)]. Live cells were stained to green with FDA (first row), and dead cells were stained to red with PI (second row). Third row (merged images of first and second rows) shows that cells cultured on polystyrene and patched by an Au-coated cover glass showed high viability, while cells patched by the ZnO and Au-coated ZnO NRAs were dead. Magnification: 4x. Scale bar: $1 \mathrm{~mm}$. 
that rapidly dividing adherent cells are more susceptible to the limited adhesion than primary cells, ZnO NRAs also promise for tumor treatment. Furthermore, taking advantage of the large surface area of a $\mathrm{ZnO}$ NRA, therapeutic chemicals can also be loaded into the $\mathrm{ZnO}$ NRA to provide a synergetic therapy. Finally, as the surface chemistry is not involved, these findings could possibly be transferred to other nanorod arrays with a similar morphology.

\section{Supplementary material}

The supplementary material for this article can be found at https://doi.org/10.1557/mrc.2018.190.

\section{Acknowledgment}

This work was partially supported by the Juvenile Diabetes Research Foundation (Award no. 2-SRA-2016-237-Q-R) and the Defense Advanced Research Projects Agency (Grant \#D17AP00031). The views, opinions, and/or findings contained in this paper are those of the authors and should not be interpreted as representing the official views or policies, either expressed or implied, of the Defense Advanced Research Projects Agency or the Department of Defense.

\section{Conflicts of interest}

The authors declare no conflicts of interest.

\section{References}

1. Z.L. Wang: Zinc oxide nanostructures: growth, properties and applications. J. Phys. Condens. Matter 16, R829 (2004).

2. T.G. Smijs and S. Pavel: Titanium dioxide and zinc oxide nanoparticles in sunscreens: focus on their safety and effectiveness. Nanotechnol. Sci. Appl. 4, 95 (2011).

3. S.N. Das, J.H. Choi, J.P. Kar, and J.M. Myoung: Tunable and reversible surface wettability transition of vertically aligned $\mathrm{ZnO}$ nanorod arrays. Appl. Surf. Sci. 255, 7319 (2009).

4. J.W. Rasmussen, E. Martinez, P. Louka, and D.G. Wingett: Zinc oxide nanoparticles for selective destruction of tumor cells and potential for drug delivery applications. Expert Opin. Drug Delivery 7, 1063 (2010).

5. P.J.P. Espitia, N.d.F.F. Soares, J.S.d.R. Coimbra, N.J. de Andrade, R.S. Cruz, and E.A.A. Medeiros: Zinc oxide nanoparticles: synthesis, antimicrobial activity and food packaging applications. Food Bioprocess Technol. 5, 1447 (2012).

6. Z.L. Wang: Nanostructures of zinc oxide. Mater. Today 7, 26 (2004).

7. J.K. Park, Y.-J. Kim, J. Yeom, J.H. Jeon, G.-C. Yi, J.H. Je, and S.K. Hahn: The topographic effect of zinc oxide nanoflowers on osteoblast growth and osseointegration. Adv. Mater. 22, 4857 (2010).

8. G. Ciofani, G.G. Genchi, and V. Mattoli: ZnO nanowire arrays as substrates for cell proliferation and differentiation. Mater. Sci. Eng., C 32, 341 (2012).

9. R.A. Brown, C.J. Barnett, A. Tarat, D.R. Jones, L.W. Francis, S. Conlan, and T.G.G. Maffeis: The viability of U-2 OS cells on zinc oxide nanowires observed via MTS assay in vitro. In Proceedings 2012 12th IEEE International Conference on Nanotechnology (IEEE-NANO), p 1.

10. S.H. Bhang, W.S. Jang, J. Han, J.K. Yoon, W.G. La, E. Lee, Y.S. Kim, J. Y. Shin, T.J. Lee, H.K. Baik, and B.S. Kim: Zinc oxide nanorod-based piezoelectric dermal patch for wound healing. Adv. Funct. Mater. 27, 1603497 (2016).

11.Z. Li, R. Yang, M. Yu, F. Bai, C. Li, and Z.L. Wang: Cellular level biocompatibility and biosafety of ZnO nanowires. J. Phys. Chem. C 112, 20114 (2008).

12. Y. Wang, Y. Wu, F. Quadri, D.J. Prox, and L. Guo: Cytotoxicity of ZnO nanowire arrays on excitable cells. Nanomaterials 7, 80 (2017).
13.J. Lee, B.S. Kang, B. Hicks, T.F. Chancellor, B.H. Chu, H.-T. Wang, B. G. Keselowsky, F. Ren, and T.P. Lele: The control of cell adhesion and viability by zinc oxide nanorods. Biomaterials 29, 3743 (2008).

14. T.D. Zaveri, N.V. Dolgova, B.H. Chu, J. Lee, J. Wong, T.P. Lele, F. Ren, and B.G. Keselowsky: Contributions of surface topography and cytotoxicity to the macrophage response to zinc oxide nanorods. Biomaterials 31, 2999 (2010).

15. R. Ning, S. Wang, J. Wu, F. Wang, and J.M. Lin: Zn0 nanowire arrays exhibit cytotoxic distinction to cancer cells with different surface charge density: cytotoxicity is charge-dependent. Small (Weinheim an der Bergstrasse, Germany) 10, 4113 (2014).

16. V. Errico, G. Arrabito, E. Fornetti, C. Fuoco, S. Testa, G. Saggio, S. Rufini, S. Cannata, A. Desideri, C. Falconi, and C. Gargioli: High-density ZnO nanowires as a reversible myogenic-differentiation switch. ACS Appl. Mater. Interfaces 10, 14097 (2018).

17. J. Bogdan, J. Pławińska-Czarnak, and J. Zarzyńska: Nanoparticles of titanium and zinc oxides as novel agents in tumor treatment: a review. Nanoscale Res. Lett. 12, 225 (2017).

18. S. Xu, C. Lao, B. Weintraub, and Z.L. Wang: Density-controlled growth of aligned $\mathrm{ZnO}$ nanowire arrays by seedless chemical approach on smooth surfaces. J. Mater. Res. 23, 2072 (2008).

19. J.-H. Tian, J. Hu, S.-S. Li, F. Zhang, J. Liu, J. Shi, X. Li, Z.-Q. Tian, and Y. Chen: Improved seedless hydrothermal synthesis of dense and ultralong Zn0 nanowires. Nanotechnology 22, 245601 (2011).

20. 0. Lupan, L. Chow, G. Chai, B. Roldan, A. Naitabdi, A. Schulte, and $\mathrm{H}$. Heinrich: Nanofabrication and characterization of $\mathrm{ZnO}$ nanorod arrays and branched microrods by aqueous solution route and rapid thermal processing. Mater. Sci. Eng., B 145, 57 (2007).

21. M. Mehrabian, R. Azimirad, K. Mirabbaszadeh, H. Afarideh, and M. Davoudian: UV detecting properties of hydrothermal synthesized Zn0 nanorods. Phys. E 43, 1141 (2011).

22. R. Pattern: Neonatal Rat Cardiomyocyte Harvest Protocol-Abcam. http:// www.abcam.com/ps/pdf/protocols/neonatal_rat_cardiomyocyte_harvest. pdf (accessed December 21, 2016).

23. K.H. Jones and J.A. Senft: An improved method to determine cell viability by simultaneous staining with fluorescein diacetate-propidium iodide. J. Histochem. Cytochem. 33, 77 (1985).

24.Y. Zhang, M.K. Ram, E.K. Stefanakos, and D.Y. Goswami: Synthesis, characterization, and applications of $\mathrm{ZnO}$ nanowires. J. Nanomater. 2012, 22 (2012).

25.J. Zhong, K. Cheng, B. Hu, H. Gong, S. Zhou, and Z. Du: Temperature-controlled growth and optical properties of $\mathrm{ZnO}$ nanorods with quadrangular and hexagonal cross sections. Mater. Chem. Phys. 115, 799 (2009).

26. J.F. Moudler, W.F. Stickle, P.E. Sobol, and K.D. Bomben: Handbook of $X$-ray Photoelectron Spectroscopy (Perkin-Elmer, Eden Prairie, MN, USA, 1992).

27. L. Jing, Z. Xu, J. Shang, X. Sun, W. Cai, and H. Guo: The preparation and characterization of ZnO ultrafine particles. Mater. Sci. Eng., A 332, 356 (2002).

28. K. Bhavsar, R. Prabhu, and P. Pollard: Investigations on surface wettability of $\mathrm{ZnO}$ nanowires using UV LEDs for biosensing applications. IOP Conf. Ser.: Mater. Sci. Eng. 64, 012033 (2014).

29. V. Khranovskyy, T. Ekblad, R. Yakimova, and L. Hultman: Surface morphology effects on the light-controlled wettability of $\mathrm{ZnO}$ nanostructures. Appl. Surf. Sci. 258, 8146 (2012). 